\title{
How to help: Can more active behavioral measures help transcend the infant false-belief debate?
}

\author{
Jedediah W.P. Allen \\ Bilkent University, Department of Psychology, Ankara 06800, Turkey
}

\section{A R T I C L E I N F O}

\section{Article history:}

Received 14 May 2015

Received in revised form

24 July 2015

Accepted 25 July 2015

Available online 6 August 2015

\section{Keywords:}

Social situations

False-belief

Mindreading framework

Action-based framework

Preschoolers

\begin{abstract}
A B S T R A C T
The use of looking time procedures for the claim that infants understand other's false-beliefs has drawn criticism. In response, Buttelmann, Carpenter, and Tomasello (2009) have argued for the use of a more active behavioral measure involving children's willingness to help others. However, the current study challenges Buttelmann et al.'s response on both theoretical and methodological grounds. Theoretically, Buttelmann et al. take a mindreading framework for granted and are thus committed to the same type of "rich" interpretations that have accompanied infant looking procedures more broadly. Methodologically, the current study challenges Buttelmann et al.'s interpretation that children were using the adult's falsebelief to determine how to help in this paradigm. To test our alternative perspective, mentalistic and non-mentalistic interpretations of preschooler's helping behavior were compared. In the original study, the adult's false-belief was conflated with the playing of a trick. When these two factors were separated, children's helping behavior was not consistent with the adult's false-belief. Second, when the situation was characterized in terms of a hiding scenario (instead of playing a trick), older children altered their helping behavior accordingly. Together, these results provided evidence that children in the activehelping paradigm did not use the adult's false-belief to determine how to help and that the broader social situation is an important variable for understanding other's actions. In conclusion, the use of more active behavioral measures alone does not resolve the controversy that has played out with respect to infant looking procedures. Instead, any adequate methodological modifications must be accompanied by theoretical considerations as well.
\end{abstract}

() 2015 Elsevier Ltd. All rights reserved.
There is growing support for the conclusion that infants possess a rudimentary understanding of other people's beliefs as representational states. The empirical basis for this conclusion comes from looking time studies indicating that infants are able to pass "age-appropriate" False-Belief (FB) tasks (Onishi \& Baillargeon, 2005; Surian, Caldi, \& Sperber, 2007). However, the ability to reason about another person's false-beliefs has traditionally been assumed to develop during the preschool years (Wellman, Cross, \& Watson, 2001). Therefore, a new theory of mind debate exists about whether these early looking methodologies involve the need to attribute false beliefs to others or not (Low \& Perner, 2012; Ruffman, Taumoepeau, \& Perkins, 2012; Stone, Carpendale, Sugarman, \& Martin, 2012). One well-developed proposal against early belief attribution offers a new theoretical perspective in which looking procedures are interpreted as only demonstrating

E-mail addresses: jallen@bilkent.edu.tr, jedediahwpallen@gmail.com. the tracking of "belief-like" states with certain signature limits (Apperly \& Butterfill, 2009; Low \& Watts, 2013).

An alternative approach - in favor of early belief attribution proposes that the creation of new methodologies can provide less controversial evidence for early false-belief understanding by avoiding the use of looking times altogether (Buttelmann, Carpenter, \& Tomasello, 2009). Specifically, Buttelmann et al. argue that "active behavioral measures should be highly relevant or even crucial" (p. 338) in order to transcend the controversy surrounding infant looking procedures. The rational underlying active action procedures is that they should involve some sort of interactive participation that is based on the child's understanding of the experimental situation (Meltzoff, 1995).

Although the creation of new, more active, methodologies is a productive addition to more passive looking procedures, the current article aims to demonstrate the sense in which these new active procedures face the same methodological challenges as looking procedures. The underlying commonality is not inherent to 
the procedures themselves, but rather, is a consequence of the researchers' theoretical openness to "rich" interpretations of data (Haith \& Benson, 1998). Infant research involving rich interpretations has tended to exclude alternative theoretical perspectives through their methodology. Specifically, through the design of studies that do not include the type of control conditions that would be motivated from within those alternative theoretical perspectives. Thus, the lack of such control conditions is a manifestation of underlying theoretical commitments that are often times not shared by opponents in what are assumed to be empirical debates (Allen \& Bickhard, 2013a; Müller \& Giesbrecht, 2008; Müller \& Overton, 1998).

In the realm of social-cognition, empirical research has tended to take a mindreading framework for granted (Andrews, 2012; Ratcliffe, 2007). Experimentally, this mindreading assumption manifests as an exploration of which mental-states are operative at what ages rather than as the testing of alternative (non-mentalistic) interpretations for how children understand others' actions (Huang, Heyes, \& Charman, 2002; Ruffman et al., 2012; Sirois \& Jackson, 2007). It is typically assumed that if children understand the meaning of a social situation as involving more than just "perceptual surface structure" (i.e., behavior), then such understanding must indicate some form of "conceptual deep structure" (i.e., mindreading, see Allen \& Bickhard, 2013b). This assumption systematically misguides experimental design such that success on a task is taken as evidence for mindreading and failure is interpreted as the inability to mindread. For example, researchers assume that if children preferentially imitate the intentional actions of an adult model ("success"), then they are engaging in some form of mindreading (Carpenter, Akhtar, \& Tomasello, 1998; Meltzoff, 1995). However, there are other perspectives on how children (and adults) can understand the intentionality of actions without engaging in any form of mental-state attribution (Carpendale \& Lewis, 2015; Gergely \& Csibra, 2003; Marken, 2002; RaczaszekLeonardi, Nomikou, \& Rohlfing, 2013). Importantly, these perspectives are not advocating for a behaviorist approach. Rather, they are non-mindreading ways of capturing how people understand the meaningfulness of intentional action. That is, they are non-mindreading ways of capturing how people represent the "conceptual deep structure". Accordingly, empirical demonstrations that children will differentially imitate intentional versus unintentional actions does not in itself indicate whether these children are engaging in mindreading.

Methodologically, taking a mindreading framework for granted means that there is minimal consideration for the possibility that task success could be based on the salient affordances of the agents/ objects involved and/or from expectations based on the broader social situation. For example, Huang et al. (2002), Huang and Charman (2005) introduced control conditions for Meltzoff's (1995) imitation study in order to test mentalistic versus nonmentalistic interpretations of infants' behavior. In these studies, they provided compelling evidence to suggest that dynamic object affordances were sufficient to explain infants' performance from the original study. Further, when the same procedure was used with 3.5-year-olds, those children violated the criteria used to conclude that 18 -month-olds were mindreading (i.e., they copied the literal actions of the adult model, see Huang, Heyes, \& Charman, 2006). This latter finding highlights the utility of extending the ageranges used for collecting data about children's performance on tasks with controversial interpretations.

The issue is not whether mindreading research has included numerous possible control conditions for a given study but whether such research has included the type of control conditions that are motivated from within a non-mentalistic perspective (Huang et al., 2002; Ruffman et al., 2012; Sirois \& Jackson, 2007).
Mindreading research often produces experimental evidence that is consistent with a mentalistic framework but does not test that stance against alternative non-mentalistic interpretations. To clarify, non-mentalistic perspectives include both empiricistbehaviorist approaches and action-based approaches.

Most of the empirical response to mentalistic interpretations comes from researchers operating from within an empiricistbehaviorist perspective. Part of the reason for this is a consequence of the influence that animal research has had on developmental psychology. However, there is a deeper connection between "rich" mentalistic interpretations and "lean" behavioral interpretations of experimental performances. Both sides of this debate are committed to the same theoretical assumption regarding the split between perceptually given behavior and perceptually hidden mentality. The "rich" mentalistic camp tends to argue that understanding meaning (through mindreading) is necessary to explain performance on a given task while the "lean" behavioral camp tends to argue that understanding behavior is enough. This is mostly a methodological debate in which "lean" researchers demand stricter methodological criteria for making mentalistic conclusions (Perner, 2010; Povinelli \& Vonk, 2003; Ruffman et al., 2012).

Perner (2010) is explicit that behavior-rules are not "mindblind"; however, it is unclear how to both avoid a mindreading framework and adequately explain the meaningfulness of action without adopting an action-based framework toward knowledge and social-understanding more broadly (Allen \& Bickhard, 2013a, b; Carpendale \& Lewis, 2006, 2015). From an action-based perspective there is no split between surface behavior and hidden mentality but that difference is not essential for methodological critique about the diagnostic adequacy of experimental design. Thus, an action-based approach and an empiricist-behaviorist approach are united in their methodological criticisms of mentalistic research while being opposed to each other with respect to theoretical and explanatory considerations (Carpendale, Atwood, \& Kettner, 2013).

The origins of the methodological differences between "rich" and "lean" interpretations can be traced back to why false-belief plays such an important role in theory of mind research. Part of the diagnostic power of the original Maxi task was that it seemed to test mentalistic and non-mentalistic perspectives (see commentaries in response to Premack \& Woodruff, 1978; Wimmer \& Perner, 1983). Specifically, the original study sought to tease apart responses based on the child's perspective from those based on Maxi's (i.e., Maxi's false representation of the situation). It is in the false-belief situation (i.e., when Maxi doesn't know that the chocolate has been moved) that these two perspectives have different implications for where Maxi should look. A further aspect of this task that has not tended to be the focus of discussion concerns the relative novelty involved. In general, the novelty of a task is going to reduce the possibility that children are generalizing from relevantly similar experiences (Andrews, 2012; Povinelli \& Vonk, 2003). Therefore, it is in the false-belief situation that we get the differential behavior of children who are reasoning about Maxi's falsebelief perspective from those who are using their own perspective.

The current point is not to argue that the Maxi task succeeds in being diagnostically definitive. Instead, the point is to illustrate that the focus on false-beliefs was derivative from concerns about diagnostic adequacy - i.e., testing mentalistic and non-mentalistic alternative interpretations. In contrast, contemporary socialcognition research tends to assume a mentalistic framework and empirical efforts are geared toward demonstrating mindreading abilities for different mental-states earlier than prior research. While the "descriptive" function of such studies is an important contribution to developmental research, such a strategy is more 
problematic for studies that are intended to help resolve controversy between mentalistic and non-mentalistic perspectives (i.e., Buttelmann et al., 2009).

In sum, mindreading research does an excellent job of devising innovative methodologies and experimental procedures that demonstrate children's varied understanding of the meaningfulness of different types of social situations. However, such research tends to explain that meaningfulness in terms of mental state attributions that abstract away from the complexity of 'social living' in everyday life (Andrews, 2012; Reddy, 2007; van Geert \& Steenbeek, 2013). The use of looking paradigms for cognitively "rich" interpretations of infant abilities involves a package of questionable theoretical commitments. Further, these commitments have manifested, methodologically, in terms of issues concerning control conditions (Allen \& Bickhard, 2013a; Haith \& Benson, 1998; Müller \& Giesbrecht, 2008; Müller \& Overton, 1998). The current article argues that the same systematically motivated exclusion of relevant control conditions is present for active action paradigms as well.

\section{Active interaction as an alternative to passive looking}

Standard looking procedures involve a passive looking preference to an unfolding visual event that proceeds independently of children's own looking behavior. In contrast, an active action procedure involves the interactive participation of children so as to reveal (through action) their understanding of the situation. The canonical example of an active action paradigm is an earlier study by Meltzoff (1995). In this study, Meltzoff used the tendency of children to imitate adults as a means of investigating the mental intention reading abilities of 18 -month-olds. Specifically, an experimenter failed at their attempts to demonstrate some "intended" actions with variuos objects (e.g., because of misalignment the experimenter failed to place a ring on a peg or to insert a block into a hole). When infants were given their turn, they tended to perform the actions that the adult model "intended" to demonstrate (e.g., they put the ring on the peg) rather than the "literal" actions that were actually demonstrated (e.g., missing the hole with the block).

In the Buttelmann et al. study, researchers used the tendency of children to help adults as a means of investigating their false-belief reasoning abilities. Specifically, children were given an opportunity to help the adult who possessed either a false belief or a true belief about the location of the adults' favorite toy. Similar to the structure of the original false-belief procedure (Wimmer \& Perner, 1983), an experimenter (E2) placed a toy into one of two identical boxes; then, a different experimenter (E1) and the child moved the toy to the other box, either in the absence of E2 (false-belief condition $\mathrm{FB}$ ), or in the presence of E2 (true-belief condition - TB). ${ }^{1}$

During the test phase, E2 returned to the room and attempted to open the box that originally contained the toy (i.e., the now-empty box). Unable to do so, the child was allowed/encouraged to help E2. At this point, children could help by opening the box that E2 had just tried to open (i.e., the now-empty box) or they could help by opening the other box with the toy inside. If children understood that E2 possessed a false-belief about the location of the toy, then they would interpret E2's effort to open the now-empty box as an attempt to retrieve the toy. Therefore, in this condition, children were predicted to help by getting the toy from the other box. In

\footnotetext{
${ }^{1}$ Notice the sense in which these labels presuppose a mentalistic interpretation of the experimental situation. It was the presence or absence of the adult that was manipulated in the Buttelmann et al. study and it was the commitment to a mindreading framework that motivated the assumption that children understood the situation in terms of the adult's beliefs.
}

contrast, in the true-belief condition, children would presumably understand that E2 wanted to open the empty box rather than retrieve the toy since E2 had witnessed the displacement of the toy into the other box.

The results from Buttelmann et al.'s first study indicated that a majority of 2.5-year-olds (75\%) in the true-belief condition helped E2 by opening the box that E2 had just tried to open (i.e., the nowempty box). In contrast, a majority of children (83\%) in the falsebelief condition retrieved the toy from the other box. These results demonstrated that children were able to correctly identify the desired goal/outcome of E2 in the test phase. Further, children understood how the desired goal/outcome related to whether E2 was present during the displacement of the toy (TB condition) or absent (FB condition). Study 2 was a replication of the first study using 16- and 18-month-olds. Results for the 18-month-olds mirrored those of the 2.5-year-olds while the results for the 16month-olds showed only partial similarity. Specifically, results for the 16-month-olds were similar to the older children in the falsebelief condition but they were at chance in the true-belief condition. Collectively, these findings were interpreted as demonstrating that "by 18 months and possibly by 16 months of age infants clearly make use of their understanding of others' false-beliefs to help them appropriately" (p. 341).

Although these results are consistent with the conclusion that infants are reasoning about the belief states of the adult in order to understand what the adult wants, and therefore how to help, there are two aspects of the results that provide reason to be skeptical of this mentalistic interpretation.

First, the pattern of results for the 16-month-olds was that they correctly selected the toy in the FB condition but were at chance in the TB condition. This pattern of results is contrary to previous empirical findings and runs counter to several developmental theories that predict that true-belief understanding emerges before false-belief understanding. That is, the results from the Buttelmann et al. study imply that true-belief understanding improves between 16- and 18-months while false-belief understanding is equally present at both ages. An alternative interpretation is that 16month-olds were preferentially retrieving the toy in the FB condition for reasons that were independent of reasoning about beliefs altogether - one plausible possibility being that the toy was relatively salient as a possible goal for E2 (Andrews, 2012).

Second, in the false-belief condition, after E2 re-entered the room (during the test phase) but before $\mathrm{E} 2$ failed to open the box that originally contained the toy, seven $(\sim 60 \%)$ of the 2.5 -year-olds spontaneously tried to inform E2 that the toy had been moved (in contrast, only 1 child did so in the TB condition). This is important because at this point in the procedure E2 had not yet failed to open the box that originally held the toy. Therefore, children should not yet be able to make the inference about what E2 wanted (recall, it is E2's failed-attempt to open the box in conjunction with knowledge about E2's false-belief that is the purported basis for interpreting E2's actions as an attempt to retrieve the toy). Consequently, something else about the experimental setting must have motivated children to "spontaneously" assume that E2 wanted the toy one likely possibility being that the toy was actually part of a broader social situation in which E1 and the child were playing a trick on E2.

The Buttelmann et al. study does not control for the possibility that the type of social situation might have constituted the relevant difference between the false-belief and true-belief conditions. Other researchers have also pointed out the potential relevance of the social nature of the interaction involved in the Buttelmann et al. study (Andrews, 2012; Doherty, 2011; Low \& Perner, 2012). From the current perspective, it is the "game" of playing a trick (i.e., hiding the toy) that defines the social situation for the child in the 
false-belief condition. Children have an extensive history of learning experiences with objects and with adults in of different types of social situations involving routines, games, and various sorts of coordination (Martin, 2012; Raczaszek-Leonardi et al., 2013). Hiding the toy in the context of playing a trick not only makes the toy particularly salient but it also creates an expectation that the adult is going to return and look for the toy. The result from Buttelmann et al.'s study indicated that as much as $60 \%$ of the children had this expectation. Therefore, from the current perspective, hiding the toy in the context of playing a trick constitutes a potentially relevant independent variable that differed between false-belief and true-belief conditions in the original study.

The more general issue regarding the paradigm used by Buttelmann et al. is that it did not include the possibility of a nonmentalistic alternative interpretation for how children understood the situation. That is, the procedure did not test for the possibility that children may have helped the adult (i.e., understood what the adult wanted) without reasoning about his/her (false) belief states. Buttelmann et al. pursued the laudable goal of providing new empirical evidence in an effort to begin to resolve the infant false-belief debate. However, such a goal involves ruling out the possibility of alternative non-mentalistic interpretations for successful performance. False-belief tasks tend to be empirically stronger tests of mindreading because they seem to require children to reason about the adult's false beliefs in order to succeed. If success on the helping task can plausibly be accomplished without reasoning about the adult's false-beliefs, then the task is empirically inadequate to differentiate between alternative interpretations. $^{2}$

\subsection{Unitary explanation across both conditions}

A possible objection regarding the adequacy of formulating a rebuttal is to argue that a successful critique must provide a single, unified, alternative account of all of the results being questioned (Baillargeon, 2000). The underlying basis for such an objection may derive from the idea that the results from different studies provide independent measurements of the ability in question. Therefore, critiquing one set of results is insufficient to rebut the conclusions about that ability. In the case of the Buttelman et al. study, this objection would mean that a successful critique ought to provide a single unified explanation for the pattern of helping behavior across both true-belief and false-belief conditions. From within the mindreading framework that the Buttelmann et al. study was designed and interpreted, belief attribution provides that unified explanation. In contrast, a non-mentalistic perspective raises the possibility that there are two different explanations for children's helping behavior in the two conditions (i.e., hiding the toy in the trick scenario for the "false-belief" condition and the action affordances/functioning of the box for the "true-belief" condition). To require a unified account of both true-belief and false-belief conditions is to beg the question concerning whether or not there is a unified explanation. Such an assumption would impose an a priori constraint on researchers that is neither empirically nor theoretically well grounded. If it is accepted as a possibility that there could be two different processes involved in the two conditions, then the "true-belief" condition from the original study would no longer have the same relevance as it did when there was only one process involved (i.e., providing a baseline for comparison with the false-

\footnotetext{
${ }^{2}$ False-belief tasks should also involve sufficient novelty in order to rule out performance that is based on previously learned heuristics for predicting others' behavior in "false-belief" situations (Andrews, 2012).
}

belief condition such that children don't always just retrieve the toy).

\section{The current study}

The current study was intended to serve several functions. First, it provides an empirical response to the findings and conclusions from the Buttelmann et al. study. However, suggesting that the trick scenario may have been a confounding variable does not, by itself, require consideration of any deeper issues. Second, it demonstrates the methodological implications of drawing our attention to the richness of the broader social situation as a source for how children understand the meaningfulness of other's actions. Csibra and Gergely (2009) have demonstrated the utility of looking at how pedagogical situations set certain parameters that influence how children will interpret human activity. The current perspective assumes that such parameter setting goes beyond pedagogy to a broad plurality of types of social situations that increasingly differentiates with development. Third, the study provides the empirical aspect of the broader explication for what it means to say that the experimental design and interpretation of the original study took a mindreading framework for granted. Fourth, the study provides some empirical warrant to the existing theoretical justification for taking an action-based perspective toward social ontology. That is, a perspective in which action-based characterizations of types of social situations defines the meaningfulness of social activity (Bickhard, 2008; Carpendale \& Lewis, 2015).

The current study used three conditions in an effort to clarify the underlying basis of children's helping behavior in this paradigm. The first condition was a replication of the false-belief condition from the original study. The second condition (the Clairvoyance condition, CV) provided a test of the false-belief interpretation in a way that did not conflate other aspects of the experimental setting (i.e., the broader social situation). Specifically, after returning to the room, E2 tried to open the box that "should have" been empty (i.e., according to his/her false belief). In this way, the clairvoyance condition separated the salience of the toy (induced by hiding the toy in the trick scenario) from the search behavior that was implied by E2's false belief. If children were interpreting E2's actions by using E2's false-belief, then that should be the case whether E2's false-belief implied that he/she wanted the toy (the false-belief condition) or an empty box (the clairvoyance condition). If children help E2 by opening the empty box, then it would suggest that they are using E2's false-belief to determine what E2 wants. If instead children help by retrieving the toy, it would suggest that a non-mentalistic interpretation of children's behavior is warranted (unless children attributed the adult with clairvoyance).

The underlying logic for this condition is the same as that of the false-belief condition from the original study. In both cases, it is assumed that children are making a goal-inference on the basis of the adult's false-belief. The difference concerns the specific content of the false-belief and the subsequent goal-inference that is implied. Although the clairvoyance condition can rule against the false-belief interpretation, it does not provide direct evidence that the type of social situation is the relevant variable guiding children's helping behavior. Therefore, the third condition (the HandsFull condition, HF) manipulated the broader scenario in order to explore whether invoking a novel social situation is sufficient to guide children's interpretation of the adult's actions in this paradigm. Specifically, the hands-full condition attempted to demonstrate how the broader social context helps define the relevance of different aspects of the situation (i.e., the relevance of the empty box changes if the adult needs to put away some toys). Therefore, as in the clairvoyance condition, the adult returns to the room and tries to open the box that had previously been empty; however, 
instead of playing a trick, a hiding scenario was used that would make wanting an empty box a potentially relevant goal for the adult. That is, in contrast to the clairvoyance condition, children now had a reason to open the other (empty) box - because the adult needed somewhere to hide what was in his/her hands.

Predictions. If children act according to the search implications that are induced by the broader social situation (as opposed to the search implications of the adult's false-belief), then children should help the adult by retrieving the toy in the clairvoyance condition and by opening the empty box in the hands-full condition.

To clarify, the methodological contrast between a mindreading perspective and a social situation perspective on the development of children's social understanding is going to concern: what aspects of the experimental situation are considered relevant; and, once those aspects have been considered, whether mental-state attributions are superfluous. In the current context, the mindreading perspective of the Buttelmann et al., study assumes that the relevant aspects of the situation are restricted to the perceptually available presence or absence of the adult (E2). From a social situation perspective, children have developed increasingly differentiated knowledge about different types of social situations and how that knowledge relates to the meaning of unfolding activity within those situations. Accordingly, the affordances of the boxes, the toy, the adult, and how those affordances change with respect to the types of social situations involved, constitutes the relevant aspects of the situation. It could be conceded that these other aspects of the social situation are the relevant aspects of the "perceptually" 3 available ground upon which belief attributions are inferred; but then, such attributions become superfluous - they don't add anything to the account. This latter point concerns more of the theoretical contrast between perspectives and will be taken up in the discussion section.

\subsection{Using preschoolers in the current study}

The experiments by Buttelmann et al. included young preschoolers (study 1) and infants (study 2). The current study included only preschoolers that ranged in age from 3 to 5 years. There are both methodological and theoretical reasons for this difference that accord with the basic challenge of the current study that the helping procedure does not require belief reasoning.

On methodological grounds, results from the original study suggest that the helping procedure may not be an age-appropriate task for infants. There are two findings by Buttelmann et al., that support this claim. First, the exclusion rate for the infant study was high. Essentially, there were equal numbers of infants who participated in the study as were excluded from the study such that the exclusion rate was the same as the sample size $(\mathrm{N}=\sim 100)$. Such a high exclusion rate raises questions about the conclusions of the study because it suggests that participants did not understand the experimental set up.

Second, as mentioned above, results from the 16-month-olds implied that they have false-belief understanding but not truebelief understanding. An alternative possibility is that these infants were drawn to the toy independently of the adult's beliefs. As the authors discuss, the 16-month-olds may have had difficulty inhibiting the desire to retrieve the toy. Without additional measures and controls, it is again difficult to interpret the results for this

\footnotetext{
${ }^{3}$ Affordances are inherently related to the activity of the agent and are not perceptually available in a passive encoding sense that is often assumed by an empiricist-behavioral approach. Further, types of social situations are not perceptually available so much as they are part of children's developmental history of patterns of interaction.
}

age group. In short, the more reliable results pertain to the 2.5-yearolds and suggest that the paradigm may not be age-appropriate for infants (at least not without some modifications). In order to help clarify how children understood the experimental situation (and the influence that such understanding has on their helping behavior), only preschool-aged children were used in the current study.

On more theoretical grounds, using 3- to 5-year-olds provided additional evidence about the role, if any, that the capacity for belief reasoning plays in the helping procedure. The design of the current study is focused on testing whether the helping procedure requires belief reasoning. If it does not require belief reasoning, then the helping procedure is diagnostically insufficient and does not help transcend the infant false-belief debate. There is little controversy about whether 5-year-olds are capable of reasoning about other people's beliefs in a canonical change of location situation. Therefore, behavioral differences (especially for the clairvoyance condition) between younger and older children would suggest that standard false-belief reasoning is relevant for how older children understand the situation (i.e., the trick scenario is not a confound and only serves a motivational function). In contrast, similar performance between younger and older preschoolers would suggest that similar processes are involved for both age groups (i.e. that children's expectations about what the adult wants is provided by the scenario and the situational constraints). It is predicted that younger and older preschoolers will not differ in terms of their helping performance across all three conditions.

\section{Methods}

\subsection{Participants}

Seventy-four (47 male, 27 female) predominantly white middle class children (aged $3-5, M=48$ months, $S D=10$ ) participated in the current study. Children were recruited through published birth announcements and the university daycare. Four children were excluded from analyses for not participating (3 would not cooperate and the 4th did not want to play a trick on E2).

\subsection{Apparatus}

Materials included a large floor cushion, small stuffed animals, and two identical wooden boxes with a plastic façade and single drawer that could be "locked" by a pin inserted into the top.

\subsection{Procedure}

All children received a warm up with the two experimenters. The study was a between-subjects design in which each child was randomly assigned to one of three conditions.

\subsubsection{False-belief condition}

This condition was a replication of Buttelmann et al. and the specifics of their procedure were followed. Once one of the experimenters (E2) left the room, the other experimenter (E1) demonstrated to the child how to unlock the boxes. After the demonstration, E2 re-entered the room and E1, E2, and the child played together with E2's favorite toy. Next, E2 put the toy in one of the boxes and left the room. While E2 was absent, E1 invited the child to play a trick on E2 by hiding the toy in the other box. Finally, $\mathrm{E} 1$ and the child returned to a cushion facing the boxes and waited for E2. The testing/helping phase began when E2 re-entered the room. E2 approached the (now) empty box and attempted to open it. Unable to do so, E2 sat down between the two boxes and waited while showing signs of disappointment, puzzlement, and 
resignation. Now the response period began and the child was allowed to approach either of the boxes in an effort to help E2. After helping E2 open one of the boxes, children were asked what they thought E2 had wanted when he/she re-entered the room.

\subsubsection{Clairvoyance condition}

This condition was identical to the false-belief condition except for the box that E2 tried to open after returning to the room. Specifically, in the false-belief condition, E2 tried to open the box that he/she thought contained the toy (i.e., the box that was actually empty); whereas, in the clairvoyance condition, E2 tried to open the box that he or she thought was empty (i.e., the box that actually contained the toy).

\subsubsection{Hands-full condition}

This condition was the same as the clairvoyance condition with two relevant exceptions. First, a hiding scenario was used instead of playing a trick. When E2 first returned to the room, E2 told E1 and the child that Oscar-the-Grouch was out in the hall taking all of the toys laying around and putting them in his garbage can. E2 then suggested that E1 and the child hide their toys and placed them in one of the boxes (counter-balanced). There were several toys to ensure that the box was completely full. E2 then left to go hide some other toys that had not been put away. While E2 was gone, E1 suggested to the child that they move the toys to the other box: "Hey [child] look, that box is empty, lets see if the toys will fit in that box over there" followed by "Wow, this is really full" after the toys were placed in the box. The second difference from the clairvoyance condition was that E2 returned with his/her hands full, Upon returning, E2 had his/her hands full of toys and (similar to the clairvoyance condition) tried to open the box that he/she thought was empty - the box that actually contained the toys.

\subsection{Coding and reliability}

A majority of children had difficulty responding to the question/memory prompts about what E2 had wanted when reentering the room and this data could not be used in the main analyses. Similar to Buttelmann et al., children were coded as selecting either the toy or the empty box in their effort to help E2 accomplish his/her goal. However, some of the children who selected the empty box in the false-belief condition were still coded as selecting the toy as the goal of E2. The reason for this was that some of the 5-year-olds seemed to be extending the trick scenario by first going to the empty box rather than ending the trick by retrieving the toy directly. These children seemed to want E2 to open and find out that the original box was empty and that the toy was gone. When originally asked by E1 to help E2, these children would do things like point to the (now) empty box and say "Open that box", or "it's not in here, let's check in the other one", or pull out the pin from the (now) empty box, back away, and say "look in there".

Accordingly, a set of formal criteria was established to decide whether children who seemed to be extending the trick scenario by going to the (now) empty box nevertheless knew what E2 wanted. First, when asked if they knew what E2 wanted, they had to correctly respond that E2 wanted the toy. Second, once permitted to help, they had to respond quickly (within $5 \mathrm{~s}$ ) indicating that they had a definitive idea about how they wanted to "help". Finally, they had to do something to indicate that they wanted E2 to be the one to discover that the box was empty (e.g., explicitly tell E2, remove the pin and step back looking at E2 expectantly, etc.). Two independent coders were in agreement that all three criteria were satisfied by a total of 6 children (three 5-year-olds, two 4 -year-olds,
\& one 3-year-old). These children were "re"-coded as selecting the toy as the goal of E2 (of the 6 children there was one 4-year-old in the clairvoyance condition and the rest were in the false-belief condition). Removal of these 6 children did not change the outcome of the statistical analyses.

\section{Results}

Two planned contrasts were conducted to test the two hypotheses. Whereas the first contrast compared the false-belief and clairvoyance conditions, the second compared the clairvoyance and hands-full conditions. For the first planned contrast, a chi-square test indicated that children's selection of the box with the toy in the false-belief condition did not differ from their selection in the clairvoyance condition $\chi^{2}(1, N=47)=0.35, p=$ n.s. Specifically, most children from both conditions selected the box with the toy $(\mathrm{FB}=84 \%, \mathrm{~N}=25 ; \mathrm{CV}=82 \%, \mathrm{~N}=22$ ). A secondary analysis indicated that there was no effect of age. Specifically, 3-, 4-, \& 5-yearolds all behaved similarly in choosing the box with the toy for both the false-belief and clairvoyance conditions.

For the second planned analysis, a chi-square test indicated that children's selection of the toy in the clairvoyance condition was significantly different from their selection in the hands-full conditions $\chi^{2}(1, \mathrm{~N}=45)=4.45, p<.05$. Specifically, although $82 \%$ of children $(\mathrm{N}=22)$ in the clairvoyance condition selected the box with the toy, this number dropped to $52 \%(\mathrm{~N}=23)$ in the hands-full condition.

To explore the nature of the difference between clairvoyance and hands-full conditions, children were split into 3-, 4-, and 5year-olds, respectively. Results indicated a clear progression from 3 to 5 years of age. Specifically, most of the 3-year-olds selected the box with the toy (88\%), with roughly half of 4 -year-olds doing so (43\%), and few 5-year-olds (25\%, see Fig. 1). Follow-up analyses indicated that the difference between clairvoyance and hands-full conditions was only significant for the 5-year-olds $\chi^{2}$ (1, $\mathrm{N}=15)=5.53, p<.05$.

In sum, whereas most children in the false-belief and clairvoyance conditions selected the box with the toy regardless of age, an age-related change was observed in the hands-full condition. Specifically, as age increased, children switched from selecting the toy to selecting the empty box.

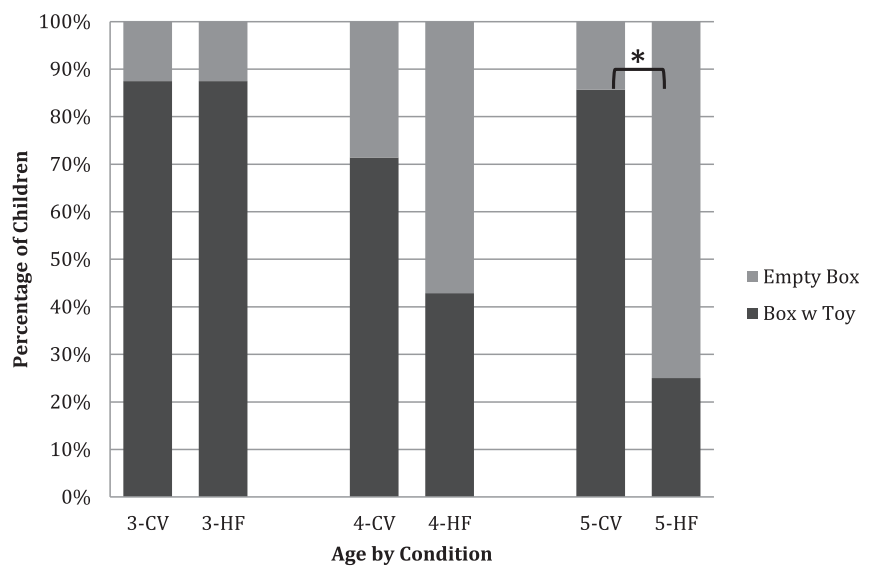

Fig. 1. Percentage of 3-, 4-, and 5-year-olds who selected the toy as their response for how to help the adult in the clairvoyance versus hands-full conditions (age means \& ranges for: $\mathrm{CV}=3.1$ years, $35-39$ months; 4.1 years, $47-51$ months; 5.1 years, $60-61$ months \& for: $\mathrm{HF}=3.1$ years, $36-37$ months; 4.0 years, $48-50$ months; 5.1 years, 59-62 months). 


\section{Discussion}

The creation of more active procedures for studying young children's mindreading abilities was intended to avoid the interpretive problems that exist for more passive looking measures. However, the current argument is that active action paradigms do not avoid the interpretive problems that exist for looking procedures. In particular, much of the controversy regarding looking paradigms derives from the theoretically motivated exclusion of relevant control conditions. Adopting a theoretical perspective is an inherent aspect of experimental research, but to help resolve debate, such perspectives should be explicitly contrasted with viable alternatives.

\subsection{The active helping paradigm}

The Buttelmann et al. study is one example of a much broader pattern of taking a mindreading perspective towards socialcognitive research for granted (Andrews, 2012; Ratcliffe, 2007). Buttelmann et al., claim that, "To pass this test children must [emphasis added] understand false-beliefs ... in order to interpret his [E2's] goal correctly and so to provide the appropriate help" (p. 338). The main methodological challenge to the active helping procedure was that children do not need to know/reason about the (false) beliefs of the adult in order to help correctly. Two experimental conditions were used to support this challenge.

First, the clairvoyance control condition was used in an effort to directly test the false-belief interpretation in a way that did not conflate other aspects of the experimental setting (i.e., the broader trick scenario as it relates to the salience of the toy). If children were using E2's false-belief to interpret his/her behavior, then they should assume that E2 wants an empty box. Results from the clairvoyance condition indicated that children consistently opened the box with the toy rather than the empty box. Therefore, the clairvoyance condition provided evidence that children were not using E2's false belief in reasoning about how to help.

Although it may be somewhat unusual for an adult to want an empty box, children in Buttelmann et al.'s original true-belief condition were willing to help the adult achieve such a goal. Further, any a priori strangeness of wanting an empty box in this situation is an indication of why the broader scenario and salience of the toy are relevant. That is, certain possibilities for what a person might want in a given situation are intrinsically much more likely and sensible than others. Determining what another person wants is always a matter of making presumptions on the basis of the available and relevant sources of information. In the current paradigm, the actions of the adult and the functioning of the objects are the potentially relevant sources of information but the meaning of both are relative to the broader social situation.

Accordingly, the hands-full condition was designed in an effort to demonstrate how the broader social situation helps define the meaning of the adult's actions. Specifically, a hiding scenario was used to make wanting an empty box a potentially relevant goal for the adult. The results indicated that changing the scenario from being about playing a trick (which makes the toy most relevant) to hiding toys from Oscar (which makes the empty box most relevant) changed how the older children understood what it could have meant to help when watching the adult try to open the (now) full box. That is, in contrast to the clairvoyance condition, the hiding scenario provided children with a reason to help the adult by opening the empty box.

The results from the hands-full condition flipped the effect that was found for the clairvoyance condition (at least for the older children). That is, except for the type of scenario, the clairvoyance and hands-full conditions were the same in terms of where the toy was located and which box the adult tried to open upon returning. That children tried to help the adult by opening the empty box demonstrated that the broader social situation can guide how older children understood what the adult might have wanted. The handsfull condition showed a developmental trend from 3- to 5-years of age such that children's preference to select the correct (empty) box increased with age. Specifically, almost all of the 3-year-olds continued to retrieve the toy in the hands-full condition despite having a good reason to assume that the adult did in fact want an empty box; 4-year-olds were at chance, while 5-year-olds correctly opened the empty box. This finding is problematic for the mindreading perspective because it demonstrates that even when there is a sensible reason for the adult to want an empty box and wanting the empty box is consistent with the adult acting on the basis of a false-belief, 3-year-olds still opened the box with the toys.

From the current perspective, the performance of 3-year-olds is less problematic. It is possible that 3 -year-olds did not understand the idiosyncrasy of the Oscar-the-grouch scenario well enough to correctly interpret the adult's actions in that specific social context. Instead, 3-year-olds may not have understood the broader social situation and may have relied on their knowledge about the conventional actions of the adult acting on the box. This possibility applies to conventional actions in general. For example, in the absence of any particular social context, if I see you pulling on a door and am asked to help, I will assume that you are trying to open the door because that is the typical function of doors when being pulled. I could be mistaken, perhaps there is a broader social situation in which a horde of Zombies are on the other side and you are trying pull the door to keep it shut. The point is that, in the absence of a broader social situation, helping someone who is acting on an object in a conventional manner only requires knowledge about the typical functioning of the object - further considerations about the mentality of the agent doing the acting appear superfluous (Andrews, 2012).

In sum, the main methodological limitation for the study by Buttelmann et al. is that it did not include control conditions for the possibility that children might be able to help the adult without utilizing some form of mindreading - e.g., by utilizing an understanding of social situations. That is, the design of the study presupposed that the only relevant theoretical considerations concerned which mental states might have been involved (e.g., true-beliefs, false-beliefs, an ignorance representation, etc.).

\subsection{Other active action paradigms}

While the methodological aspects of the current rebuttal are focused on Buttelmann et al.'s. procedure, the theoretical aspects apply more broadly. Other active action paradigms associated with the Buttelmann et al. study share the assumption that infants interpret others' actions through mindreading and then proceed methodologically on the basis of that taken-for-granted assumption. In a study by Southgate, Chevallier, and Csibra (2010), 17month-old infants were found to resolve referential ambiguity differently depending on an adult's presence or absence. During a warm-up phase, two objects were placed in two boxes and infants practiced retrieving the one that was requested by an experimenter (E1). Next, E1 left the room and a second experimenter (E2) played a trick by switching the objects that were in the boxes. At test, E1 pointed to the box that he or she thought contained a particular object in order to request it, but the majority of children went to the other box. These results were interpreted as evidence that infants used E1's false-belief about the object's location to resolve the ambiguous reference.

While an empirical response to their specific findings would be different from that of the Buttelmann et al. study, the Southgate et al. 
study encounters the same basic issue of whether children could determine what the adult was referencing without mindreading. Accordingly, that study did not test mentalistic and non-mentalistic alternative interpretations; rather, it established that infants know about some of the implications of an adult's perceptual access for resolving referential ambiguity in a joint-attention situation. Earlier research has demonstrated that, over the course of the second year, children are learning about the relevance of an adult's visual access for both the adult's search behavior and for determining reference in joint attention situations (Moll, Carpenter, \& Tomasello, 2007; Sodian \& Thoermer, 2008). In the Southgate et al. study, when the adult requested the object that was previously in one of the boxes, the adult was invoking a past situation that involved a mutually understood joint reference. This past situation of joint reference to the object was necessary in order to establish the same joint reference in the test situation. However, to claim that such re-invocations of past joint attention situations require mindreading is a theoretical issue that should be explicitly argued - not taken for granted in the design and interpretation of empirical research.

In another related study, Knudsen and Liszkowski (2012) investigated toddler's spontaneous helping behavior. These researchers used a similar change of location procedure to see if children were able to anticipate what an adult wanted before that adult had attempted any sort of action (i.e., as soon as the adult re-entered the room). Results from their study indicated that toddlers helped the adult differently depending on whether the adult had been present or absent for the displacement of an object from one container to a different one. The focus of this study was on whether children would spontaneously help the adult. In order to know how to help the adult, toddlers needed to have previously established what the adult was going to want when they returned to the room. In order for toddlers to establish an expectation about what the adult was going to want, the procedure had a "context phase" (i.e., a social situation). It was during their prior experience with the adult in the context phase that toddlers learned what was supposed to happen (i.e., the normative structure of the game was established). This normative structure was necessary for children to anticipate the adult's "mistaken" action, but, contrary to a mindreading perspective, it was also sufficient (i.e., the additional step of attributing to the adult a mental goal and false-belief is possible but unnecessary).

This study demonstrated that children as young as 18-months can learn about a relatively new social situation involving search behavior that can then form the basis of subsequent proactive helping. The finding that children do establish expectations about what the adult is likely to want based on his or her actions in a prior social situation also provides evidence for the current challenge against the Buttelmann et al., study. Specifically, that children in the false-belief condition already knew what the adult wanted when he/she re-entered the room. In the Buttelmann et al. study, it is only after $\mathrm{E} 2$ attempted to open the box that children are supposed to be able to make the purported inference about what the adult wants.

In sum, by proactively helping the adult who was not present for the displacement of the object, children showed that they knew something about the relevance of visual access for playing the normatively structured game. Although this is consistent with the further claim that children are reasoning about the adult's falsebeliefs, the conditions of the study did not allow the authors to test this claim.

The broader issue for the Knudsen \& Liszkowski study is that it makes the assumption that action anticipation must involve mindreading:

Predicting others' actions is based on 'theory-of-mind' skills (Premack \& Woodruff, 1978) which are most evident in the case of predicting others' action mistakes (Dennett, 1978).
Cognitively, the prediction of action mistakes requires both an understanding of a person's [mental] goal prior to her acting ('prior intentions'; Searle, 1983; ...); and an understanding of her incorrect representation of reality [false-belief] ... Both of these mental attributions are necessary and neither of them alone is sufficient to predict a mistaken action. (p. 113)

In contrast to the above assumption, the regularity of behavior makes clear that actions can be anticipated without mindreading (Ruffman et al., 2012). Further, knowledge about cultural norms, object functioning, and occupations provide necessary and sufficient information for making certain types of action predictions (Andrews, 2012; Ratcliffe, 2007). For some examples: my anticipation that all of the cars I pass will drive on the left side of the road is grounded in the knowledge I have about driving laws; my prediction that the person with the saw will cut the wood is grounded in my knowledge about object functioning; and my expectation that the administrative assistant will answer the phone is based on my knowledge about that occupation. More crucially, to assume that action anticipation ("mistaken" or not) requires mindreading means that correct action anticipation will be interpreted as demonstrating that infants are mindreading, and incorrect anticipation will be interpreted as demonstrating the failure to mindread. Consequently, from this perspective, there is no option in which infants might correctly anticipate the adults' actions without mindreading.

In sum, the two studies just reviewed (Knudsen \& Liszkowski, 2012; Southgate et al., 2010), along with the Buttelmann et al. study, all assume that success on their tasks require mindreading. While there are theoretically plausible arguments that could make such an assumption reasonable, there are equally plausible arguments against any such assumption. Regardless, these theoretically plausible arguments were not presented; instead, a mindreading perspective is being taken for granted in the design and interpretation of the experimental conditions. If these studies are going to help resolve the infant false-belief debate, there needs to be greater clarity on the way that theoretical commitments are motivating the design and interpretation of experiments. Such theoretical clarity will facilitate the methodological goal of including control conditions that can test for alternative interpretations.

In the current context this means testing for the theoretically plausible possibility that infants can successfully help an adult or resolve referential ambiguity without reasoning about that person's (false) beliefs. Such efforts are complicated by the fact that infants seem to be able to track the behavior of people who have false-beliefs; nonetheless, findings do not demonstrate that the basis for such tracking is in fact other people's false-beliefs (Andrews, 2012). Other attempts to reconcile findings from infant studies have given rise to the idea that there are two "mindreading" systems according to which early false-belief tasks do not involve explicit belief attribution processes (Apperly \& Butterfill, 2009; Low \& Perner, 2012). However, if the current arguments and analyses are correct, social-cognition research will need to consider more closely the relevance of the broader social situation for children's developing understanding of others and their actions. In particular, experimental procedures will need to consider children's prior knowledge about the interactive affordances of the agents and objects involved in different types of social situations.

\subsection{Asymmetric burden of proof}

Tied up with methodological issues concerning the adequacy of experimental design to demonstrate mindreading conclusions are assumptions about burden of proof. In particular, there is an 
asymmetry in terms of ruling against mentalistic versus ruling against non-mentalistic alternatives: Non-mentalistic renderings of a social situation are always necessary and researchers attempt to demonstrate that they are also sufficient to explain performance. In contrast, mentalistic renderings of a social situation are always sufficient and researchers attempt to demonstrate that they are also necessary to explain performance. Part of the reason for the asymmetry is that there are always going to be mental states potentially corresponding to reality states (i.e., mental states have contents) but not the other way around (i.e., not everything is known by all people), which is why false-beliefs, accidents, and failed-attempts provide such an important experimental context.

Further, given that a mindreading approach is not advocating for a sixth sense, mindreading inferences must be grounded in some sort of prior knowledge about physical and social 'objects' in different types of situations. Accordingly, the challenge for mindreading researchers is to demonstrate that the mindreading inferences are not superfluous. That is, once the epistemic grounds for the purported mindreading inferences have been established, there must be some unique consequences for those additional mindreading inferences. Without such additional consequences, the mindreading interpretation succumbs to a "cognitive collapse" argument in which the purported mindreading inferences are superfluous for explaining performance (Perner, 2010; Povinelli \& Vonk, 2003).

A broad research program that evolved out of a version of the "cognitive collapse" argument comes from the pioneering work of Gergely and Csibra (2003). Having recognized the redundancy of a mentalistic rendering of their experimental situations that involved goal-directedness, they developed the teleological stance:

In fact, the central insight that has guided our theorizing about the functional viability of a non-mentalistic teleological stance was the realization that when taking the mentalistic stance the rationality principle is always applied to the contents that the actor's mental states represent, and not to the intentional mind states themselves. (p. 289)

The "contents" in the above quote are the perceptually available aspects of reality involved in understanding goal-directedness: situational constraints, outcome states, and actions. Although each of these aspects of reality has potential corresponding mental states (beliefs, mental goals, \& intentions to act, respectively), Gergely and Csibra's insight was that the corresponding mental states were a superfluous component of their teleological explanation. Consequently, the telelogical stance provides a robust theoretical alternative for how children can understand the meaningfulness of goal-directed behavior without needing to attribute mental goals to the agents of such behavior. More broadly, this research demonstrates that the presupposed contrast between, perceptually available surface behavior and conceptually hidden mental states, is a false divide. Instead, young children's ability to understand the meaningfulness of behavior can be grounded directly in "perceptually" available contents. Theoretical modeling efforts like the telelogical stance help researchers to transcend the presupposed metaphysical split between the mechanical/behavioral world of physical objects and the meaningful/intentional world of psychological persons.

\section{Conclusion}

6.1. Can more active behavioral measures help transcend the infant false-belief debate?

The theoretically motivated exclusion of relevant control conditions from experimental design is not specific to active action paradigms. Cognitively rich interpretations of looking paradigms have only been possible given the lack of relevant control conditions. Buttelmann et al. may be correct that "active behavioral measures should be highly relevant - or even crucial" (p. 338) for settling the debate concerning infant false-belief understanding; however, we may also need concurrent theoretical progress in order to transcend the issues that make rich interpretations so problematic in the first place.

A first step toward such theoretical progress could involve more explicit discussions about how experimental design and interpretation are tied to theoretical commitments and assumptions. Further, if the commitments and assumptions of the folkpsychological framework no longer make it the "best game in town", then perhaps we should find a new game. An action-based framework offers a robust theoretical alternative to the folkpsychology framework (Allen \& Bickhard, 2013a, b; Carpendale \& Lewis, 2006; 2015). From an action-based perspective, socialcognitive development is a matter of children learning how to participate in different types of social situations (Allen \& Ilgaz, 2016).

Action paradigms require children to be active participants in the co-constructed social reality being used to investigate their development. This captures children's stake in the interaction and allows for greater attention, and possible manipulation, of their social goals and motivations. In general, action paradigms have greater ecological validity than looking time procedures, which narrowly focus on surprise and expectation. In these senses, action paradigms would seem well suited to an action-based perspective. However, an action-based implementation of action paradigms would require a theoretical shift in perspective on the nature of social understanding. Specifically, a shift from mental state attributions to how children represent the interactive affordances of object and people in different types of social situations.

\section{Acknowledgments}

Many thanks to Mark Bickhard, Hande Ilgaz, and the anonymous reviewers for important comments on an earlier draft of the manuscript. Also, thank you to Hande Ilgaz, Andrea Lucas, Mitchell Harvey, and Bryan Hughes for their help conducting the study.

\section{References}

Allen, J. W. P., \& Bickhard, M. H. (2013a). Stepping off the pendulum: why only an action-based approach can transcend the nativist-empiricist debate. Cognitive Development, 28, 96-133.

Allen, J. W. P., \& Bickhard, M. H. (2013b). The pendulum still swings. Cognitive Development, 28, 164-174.

Allen, J. W. P., \& Ilgaz, H. (2016). Social meta-learning: Learning how to make use of others as a resource for further learning.

Andrews, K. (2012). Do apes read minds? Cambridge, MA: MIT Press.

Apperly, I. A., \& Butterfill, S. A. (2009). Do humans have two systems to track beliefs and belief-like states? Psychological Review, 116, 953-970.

Baillargeon, R. (2000). Reply to Bogartz, Shinskey and Schilling; Schilling; and Cashon and Cohen. Infancy, 1, 447-462.

Bickhard, M. H. (2008). Social ontology as convention. Topoi, 27, 139-149.

Buttelmann, D., Carpenter, M., \& Tomasello, M. (2009). Eighteen-month-old-infants show false belief understanding in an active helping paradigm. Cognition, 11, $337-342$.

Carpendale, J. I. M., Atwood, S., \& Kettner, V. (2013). Meaning and mind from the perspective of dualist versus relational worldviews: implications for the development of pointing gestures. Human Development, 56, 381-400.

Carpendale, J. I. M., \& Lewis, C. (2006). How children develop social understanding. Malden, MA: Blackwell Publishing.

Carpendale, J. I. M., \& Lewis, C. (2015). The development of children's understanding of social interaction. In S. Robson, \& S. F. Quinn (Eds.), The Routledge international handbook of young children's thinking and understanding (pp. 123-132). New York: Routlege.

Carpenter, M., Akhtar, N., \& Tomasello, M. (1998). Fourteen- through 18-month-old infants differentially imitate intentional and accidental actions. Infant Behavior 
\& Development, 21, 315-330.

Csibra, G., \& Gergely, G. (2009). Natural pedagogy. Cell Press, 148-153.

Doherty, M. J. (2011). A two systems theory of social cognition: engagement and theory of mind. In J. Roessler, H. Lerman, \& N. Eilan (Eds.), Perception, causation and objectivity (pp. 305-323). Oxford, NY: Oxford University Press.

Gergely, G., \& Csibra, G. (2003). Teleological reasoning in infancy: the naïve theory of rational action. Trends in Cognitive Science, 7, 287-292.

Haith, M. M., \& Benson, J. B. (1998). Infant cognition. In W. Damon, D. Kuhn, \& R. Siegler (Eds.) (5th ed.,Cognition, perception, and language development: Vol. 2. Handbook of child Psychology (pp. 199-254). New York: Wiley.

Huang, C. T., \& Charman, T. (2005). Gradations of emulation learning in infants' imitation of actions on objects. Journal of Experimental Child Psychology, 92, 276-302.

Huang, C. T., Heyes, C., \& Charman, T. (2002). Infants' behavioral reenactment of "failed attempts": exploring the roles of emulation learning, stimulus enhancement, and understanding of intentions. Developmental Psychology, 38, $840-855$.

Huang, C. T., Heyes, C., \& Charman, T. (2006). Preschoolers' behavioral reenactment of "failed attempts": the roles of intention-reading, emulation and mimicry. Cognitive Development, 21, 36-45.

Knudsen, B., \& Liszkowski, U. (2012). Eighteen- and 24-month-old infants correct others in anticipation of action mistakes. Developmental Science, 15, 113-122.

Low, J., \& Perner, J. (2012). Implicit and explicit theory of mind: state of the art. British Journal of Developmental Psychology, 30, 1-30.

Low, J., \& Watts, J. (2013). Attributing false beliefs about object identity reveals a signature blind spot in humans' efficient mind-reading system. Psychological Science, 24, 305-311.

Marken, R. S. (2002). Looking at behavior through control theory glasses. Review of General Psychology, 6, 260-270.

Martin, J. (2012). Coordinating with others: outlining a pragmatic perspectival psychology of personhood. New Ideas in Psychology, 30, 131-143.

Meltzoff, A. N. (1995). Understanding the intentions of others: re-enactment of intended acts by 18-month-old children. Developmental Psychology, 31, $838-850$.

Moll, H., Carpenter, M., \& Tomasello, M. (2007). Fourteen-month-olds know what others experience only in joint engagement. Developmental Science, 10, $826-835$.

Müller, U., \& Giesbrecht, G. (2008). Methodological and epistemological issues in the interpretation of infant cognitive development. Child Development, 79, 1654-1658.

Müller, U., \& Overton, W. F. (1998). How to grow a baby: a reevaluation of imageschema and Piagetian action approaches to representation. Human Development, 41, 71-111.
Onishi, K. H., \& Baillargeon, R. (2005). Do 15-month-old infants understand false beliefs? Science, 308, 255-258.

Perner, J. (2010). Who took the cog out of cognitive science. In P. A. Frensch, \& R. Schwarzer (Eds.), Cognition and neurospsychology: International perspectives on psychological science (Vol. 1, pp. 241-262). Hove, UK: Psychology Press.

Povinelli, D. J., \& Vonk, J. (2003). Chimpanzee minds: suspiciously human? TRENDS in Cognitive Science, 7, 157-160.

Premack, D., \& Woodruff, G. (1978). Does the chimpanzee have a theory of mind? Behavioral Brain Sciences, 1, 515-526.

Raczaszek-Leonardi, J., Nomikou, I., \& Rohlfing, K. J. (2013). Young children's dialogical actions: the beginnings of purposeful intersubjectivity. IEEE Transactions on Autonomous Mental Development, 5, 210-221.

Ratcliffe, M. (2007). Rethinking commonsense psychology: A critique of folk psychology, theory of mind and simulation. Palgrave Macmillan.

Reddy, V. (2007). Getting back to the rough ground: deception and 'social living' Philosophical Transactions of the Royal Society B, 362, 621-637.

Ruffman, T., Taumoepeau, M., \& Perkins, C. (2012). Statistical learning as a basis for social understanding in children. British Journal of Developmental Psychology, 30 87-104.

Sirois, S., \& Jackson, I. (2007). Social cognition in infancy: a critical review of research on higher order abilities. European Journal of Developmental Psychology 4, 46-64.

Sodian, B., \& Thoermer, C. (2008). Precusors to a theory of mind in infancy perspectives for research on autism. The Quarterly Journal of Experimental Psychology, 61, 27-39.

Southgate, V., Chevallier, C., \& Csibra, G. (2010). Seventeen-month-olds appeal to false beliefs to interpret others' referential communication. Developmental Science, 13, 907-912.

Stone, J. E., Carpendale, J. I. M., Sugarman, J., \& Martin, J. (2012). A Meadian account of social understanding: taking a non-mentalistic approach to infant and verbal false belief understanding. New Ideas in Psychology, 30, 166-178.

Surian, L., Caldi, S., \& Sperber, D. (2007). Attribution of beliefs by 13-month-olds. Psychological Science, 18, 580-586.

van Geert, P., \& Steenbeek, H. (2013). (P) (re) presentations: What are they and how do they develop? Commentary on "Stepping off the pendulum: Why only an action-based approach can transcend the nativist-empiricist debate" by J. Allen \& M. Bickhard. Cognitive Development, 28, 138-143.

Wellman, H. M., Cross, D., \& Watson, J. (2001). Meta-analysis of theory-of-mind development: the truth about false belief. Child Development, 72, 655-684.

Wimmer, H., \& Perner, J. (1983). Beliefs about beliefs: representation and constraining function of wrong beliefs in young children's understanding of deception. Cognition, 13, 103-128. 